ReBEn, 35 : 48-59, 1982

\title{
O PACIENTE CIRÚRGICO: SUAS EXPECTATIVAS E OPINIÕeS QUANTO AO CUIDADO DE ENFERMAGEM NO PERIODO TRANSOPERATÓRIO
}

\author{
Estela Regina Ferraz * \\ Orientador: Sônia Della Torres Salzano **
}

\begin{abstract}
\begin{tabular}{|c|c|}
\cline { 2 - 2 } & $\operatorname{ReBEn} / \mathrm{n5}$ \\
\hline
\end{tabular}
FERRAZ, E.R. e Orientador - O Paciente Cirúrgico: suas Expectativas e Opiniōes Quanto ao Cuidado de Enfermagem no Período Transoperatório. Rev. Bras. Enf.; DF, $35: 48-59,1982$.
\end{abstract}

\footnotetext{
O paciente cirúrgico, ao ser hospitalizado, traz consigo todas as ansiedades de uma indefinição dos fatos que irão advir. Entrará num ambiente fechado, com pessoas vestindo uniformes estranhos. Mesmo que a cirurgia seja considerada, pela equipe cirúrgica, como algo pequeno, na mente do paciente sempre será de maior importância - é a sua operação, o seu corpo, sua família se preocupará.

$\mathrm{Na}$ tentativa de levantar as reações do paciente frente à cirurgia, vários autores encontraram o medo, traduzido peir medo do desconhecido, da dor de estar dependente, da morte, receio de não ser operado pelo seu médico. LEViNE \& FIELDER (1970); GRAHAM \& MYERS (1971); KATZ et alii (1971); KAMYAMA (1972); DUARTE (1976); JOHNSTON \& HOOD (1976); BALBDA (1977); MENEZES (1978).
}

A equipe cirúrgica deve estar alertada para este fato e procurar evidenciar o grau de ansiedade do paciente. ramente dependente da enfermagem O paciente no centro cirúrgico é inteiele será anestesiado, estará incapacitado de se autocuidar. A enfermeira de centro cirúrgico é seu representante perante esta situação; deverá ter em mente que o paciente é uma pessoa que tem sus vida e sua família ansiosa à espera dos acontecimentos, membro de uma sociedade e, em parte, produto de sua estrutura.

A enfermeira de centro cirúrgico muitas vezes permanece fechada em sua unidade, envolvida em problemas técnico-administrativos, impedindo-a de conhecer os pacientes que permanecerão sob seus cuidados. Vários autores estão de acordo que a enfermagem de centro

* Auxiliar de Ensino na disciplina Enfermagem em Centro Cirúrgico - EEUSP.

* Professor Assistente Doutor na disciplina Enfermagem em Centro Cirúrgico - EEUSP. 
FERRAZ, E.R. e Orientador - O Paciente Cirúrgico: suas Expectativas e Opiniōes Quanto ao Cuidado de Enfermagem no Período Transoperatório. Rev. Bras. Enf.; DF, 35 : 48-59, 1982.

cirúrgico não poderá prestar uma assistência global ao paciente se não o conhecer antes da cirurgia e planejar sua assistência. CLEMONS (1968); GINSBERG (1969); RADINA (1969); EDVARDS (1971); GRUENDEMANN (1971); ATKINSON (1972); MEHAFFY (1975); PANZA (1977); JOUCLAS (1977); CASTELLANOS (1978).

Em trabalho realizado por MENEZES (1978), quanto à problemática de enfermagem dos pacientes no periodo transoperatório, são apresentadas as conclusões que as expectativas dos pacientes quanto à enfermagem eram relacionadas à área expressiva ou básica de atuação, isto é, esperavam o diálozo, serem observados e receberem bons cuidados.

Neste estudo, teve-se por objetivos:

1. Verificar, no período pré-operatório, quais são as expectativas do paciente em relação ao atendimento de enfermagem no centro cirúrgico;

2. Verificar no $10^{\circ}$ ou $2 .^{\circ}$ dia pós-operatório, a opinião do paciente quanto ao atendimento de enfermagem no período transoperatório;

3. Identificar os problemas sentidos pelo paciente, nos periodos pré e pós-operatórios, baseados nas entrevistas realizadas.

\section{MATERIAL E MÉTODO}

1. População: A população foi constituída de 44 pacientes, entre 20 e 60 anos, de ambos os sexos, que se submeteram a cirurgias eletivas, sob anestesia geral ou raquidiana. Eram brasileiros natos, conscientes.

2. Coleta de Dados: O levantamento foi realizado no período de 14 de junho a 2 de agosto de 1980. Utili- zou-se o formulário (anexo I) constituído de 3 partes: identificação, levantamento da opinião do paciente no periodo pré-operatório e levantamento da opinião do paciente no periodo pós-operatório.

No pré-operatório, procurou-se levantar os dados quanto ao conhecimento do paciente, cuanito à cirurgia e o que ele espera encontrar no centro cirúrgico. No pós-operatório, coletaram-se as opiniōes quanto aos eventos transcorridos no período transoperatório.

Os pacientes forain escolhidoj ao acaso na lista de cirurgia eletiva, segundo os critérios estabelecidos para amostra da população. Os dados de identificação foram obtidos no prontuário. Fezse a entrevista com o paciente no período pré-operatório (12-24 horas antes da cirurgia) e depois no pós-operatório (1. ${ }^{\circ}$ ou $2 .^{\circ}$ dia).

3. Análise Estatistica: Foi feita de modo essencialmente qualitativo, baseando-se em indices percentuais.

\section{RESULTADOS E DISCUSSĀO}

\section{Carasterísticas da amostra.}

Os resultados cbtidos mostraram que a população estudada foi equitativa na distribuição quanto ao sexo, predominantemente de estado civil casado (75\%); com instrução de nível de $1 .^{\circ}$ grau incompleto $(52,3 \%)$; ocupando funções manuais especializadas $(45,5 \%)$ como pintor, costureira etc.; como predominância da faixa etária de $40-60$ anos $(68,2 \%)$.

Os pacientes foram submetidos com grande predominância à anestesia geral (84,1\%); com períodos de duração de 3 a 4 horas $(36,4 \%)$. Verificou-se a ligeira prevalência de colecistectomia com 
FERRAZ, E.R. e Orientador - O Paciente Cirúrgico: suas Expectativas e Opiniōes Quanto ao Cuidado de Enfermagem no Periodo Transoperatório. Rev. Bras. Enf.; DF, 35 : 48-59, 1982.

exploração de vias biliares $(18,2 \%)$ em relação aos demais tipos de cirurgia realizadas.

2. Expectativas dos pacientes colhidas no período pré-operatório, quanto à assistência de enfermagem no transoperatório.

Os rasultados mostram que $\mathbf{7 9 , 5 \%}$ dos pacientes sabiam que iriam se submeter a uma cirurgia.

As respostas obtidas, quanto ao conhecimento dos pacien'tes a respeito do tipo de cirurgia a que iriam se submeter, foram agrupadas da seguinte forma:

- local da cirurgia: quando os pacientes mencionaram o local onde seria realizada a cirurgia, por exemplo: "veia carótida, rosto";

- doença que o acometera: quando os pacientes mencionaram a doença $\mathrm{e}$ pela qual foi indicada a cirurgia, por exemplo: "úlcera, falha na circulação da perna";

- tipo de cirurgia: quando os pacientes mencionaram qual o tipo de cirurgia a que se submeteriam, por exemplo: "simpatectomia";

- benefício da cirurgia: quando os pacientes mencionaram o que acarre, taria de benefício após a cirurgia, por exemplo: "melhorar a circulação do sangue."

\section{TABELA 1}

Número e porcentagem de pacientes, segundo o seu conhecimento quanto à cirurgia a ser realizada.

\begin{tabular}{l|c|c}
\hline \hline Conhecimento QUnNTo A CIRURGia & Ne & \multicolumn{1}{c}{ Porcentagem (\%) } \\
\hline - Local da cirurgia & 21 & 47,3 \\
- doença que o acometera & 14 & 31,8 \\
- tipo de cirurgia & 5 & 11,4 \\
- beneficio esperado da cirurgia & 4 & 9,1 \\
\hline \\
T O T A I & 44 & 99,6 \\
\hline \hline
\end{tabular}

Observa-se rue $47,3 \%$ dos pacientes conheciam o iocal da cirurgia a ser realizada. Os pacientes deveriam conhecer sobre sua cirurgia pois GILLIS (1962) cita que os pacientes sobrevivem às mudanças desde que elẹs conheçam e saibam que são temporárias e necessárias, ajustando-se mental e fisicamente.
Somente $40,9 \%$ dos pacientes receberam orientação sobre a cirurgia e todos afirmaram ter sido pelo médico.

As dúvidas levantadas, geralmente estavam relacionadas com o medo do desconhecido, poderiam estar aumentadas por não conhecerem ninguém no centro cirúrgico. 
F'ERRAZ, E.R. e Orientador - O Paciente Cirúrgico: suas Expectativas e Opiniōes Quanto ao Cuidado de Enfermagem no Período Transoperatório. Rev. Bras. Enf.; DF, 35 : 48-59, 1982.

TABELA 2

Número e porcentagem de pacientes, segundo a expectativa quanto à assistência de enfermagem.

\begin{tabular}{c|c|c}
\hline$E \times P E C T$ A T I A & Número & Porcentagem(\%) \\
\hline - área instrumental (*) & 37 & 84,1 \\
- área expressiva (**) & 3 & $-6,8$ \\
• näo sabiam & 4 & 9,1 \\
& & \\
\hline T O T A L & 44 & 100,0 \\
\hline \hline
\end{tabular}

Verifica-se que $84,1 \%$ dos pacientes esperavam que a enfermagem executasse atividades técnicas da área instrumental. Porém, 91,6\% dos pacientes relataram que gostariam que alguma pes- soa familiar permanecesse ao seu lado no periodo transoperatório.

Dos pacientes, $75 \%$ disseram que conheciam a enfermeira da unidade de internação, apesar de a confundirem com auxiliar $\theta$ atendente de enfermagem.

\section{TABELA 3}

Número e porcen'tagem de perguntas formuladas pelos pacientes no final da entrevista.

\begin{tabular}{|c|c|c|}
\hline PERGUNTAS FORMULADAS & Número & Porcentagem $(\%)$ \\
\hline $\begin{array}{l}\text { - média de perinanéncia no hospital } \\
\text { - horário de visita de ramiliares } \\
\text { no póz-operatório. } \\
\text { - necessidade de sonda gástrica } \\
\text { - permanência de familiares no cen } \\
\text { tro cirúrgico } \\
\text { - necessidade de retirar prótese } \\
\text { - tempo de duração da ancstesia } \\
\text { - alimentaça via oral } \\
\text { - possibjiciade de colostomia } \\
\text { Tot A L }\end{array}$ & $\begin{array}{l}1 \\
1 \\
1 \\
1 \\
1 \\
1 \\
11\end{array}$ & $\begin{array}{r}9.1 \\
9.1 \\
9.1 \\
9.1 \\
9.1 \\
100,0\end{array}$ \\
\hline
\end{tabular}

Pode-se concluir que a maior preocupação relatada pelos pacientes foi quan'to à média de permanência no hospital $(27,2 \%)$.

* Equipamentos, materiais e ordem na sala de operaçōes para os pacientes serem logo operados, anestesiados e tudo ocorresse bem.

** Ter gente amiga para ajudar. 
FERRAZ, E.R. e Orientador - O Paciente Cirúrgico: suas Expectativas e Opiniōes Quanto ao Cuidado de Enfermagem no Período Transoperatório. Rev. Bras. Enf.; DF, 35 : 48-59, 1982.

TABELA 4

Númøro e porcentagem de pacientes segundo orientações recebidas no momento da entrevista.

\begin{tabular}{|c|c|c|}
\hline ORIENTAÇOES QUANTO A & Ne & Porcentagem $(\%)$ \\
\hline - cirurgia & 14 & 63,6 \\
\hline - estar longe da familia & 3 & 13,6 \\
\hline - ancstesia & 2 & 9,1 \\
\hline - näo aceitaçāo da cirurgia & 2 & 9,1 \\
\hline - planta fisica do centro cirúrgico & 1 & 4,6 \\
\hline$T \circ T \wedge L$ & 22 & 100,0 \\
\hline
\end{tabular}

Como se pode observar, $63,6 \%$ dos pacientes que receberam orientação foram quanto à cirurgia.

Os resultados obtidos permitem inferir que há falta de orientação dos pacientes no periodo pré-oparatório quanto ao transoperatório. Observou-se que a falta de trabalho em equipe, tanto na unidade de internação como no centro cirúrgico, pode levar a um aumento da ansiedade do paciente, pois as informações não são levadas a ele.
3. Opiniōes dos pacientes, colhidas no periodo pós-operatório, quanto à assistência de enfermagem prestada no transoperatório.

Os resultados obtidos mostram que o recebimento do paciente no centro cirúrgico foi feito por funcionários em $33, \%$ dos casos. Em $61,4 \%$ dos pacientes permaneceram no corredor esperando para entrar na sala de operações e relataram que estavam calmos.

\section{TABELA 5}

Número e porcentagem de pacientes, segundo orientaçōes recebidas pacientes no período pré-operatório imediato.

\begin{tabular}{|c|c|c|}
\hline $\begin{array}{l}\text { ATIVIDADES PERCEBIDAS } \\
\text { No PRE-OPERATORIO IMEDIATO }\end{array}$ & Ne & Porcentasem (\%) \\
\hline $\begin{array}{l}\text { - passagen da maca para a mesa } \\
\text { - ser anestesiado } \\
\text { - preparo pré-operatório da pele } \\
\text { - colocaçào do soro } \\
\text { - colocação de gorro na cabeça } \\
\text { - preparo do instrumental cirurgico } \\
\text { - tirar a roupa } \\
\text { - colocaço de sonda vesical } \\
\text { - perguntas sobre jejum } \\
\text { nāo lembram }\end{array}$ & $\begin{array}{r}24 \\
15 \\
8 \\
4 \\
2 \\
1 \\
1 \\
1 \\
1 \\
10\end{array}$ & $\begin{array}{r}35,8 \\
22,4 \\
11,9 \\
6,0 \\
3,0 \\
1,5 \\
1,5 \\
1,5 \\
1,5 \\
14,9\end{array}$ \\
\hline 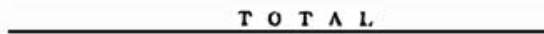 & 67 & 100.0 \\
\hline
\end{tabular}


FERRAZ, E.R. e Orientador - O Paciente Cirúrgico: suas Expectativas e Opiniōes Quanto ao Cuidado de Enfermagem no Período Transoperatório. Rev. Bras. Enf.; DF, 35 : 48-59, 1982

DE LAPPE (1974) declara que para o início de comunicação com o paciente no centro cirúrgico, a equipe deve "dirigir-se a ele, cumprimentá-lo e chamá-lo pelo nome, apresentando-se como um profissional disposto a diminuir suas tensōes". Dos pacientes, $77,3 \%$ foram tratados pelo nume.

Verifica-se que $35,8 \%$ dos pacientes relataram como atividade no centro cirúrgico a passagem da maca para a mesa cirúrgica. Essas atividades perce- bidas são todas da área instrumental de atuação.

Antes da cirurgia, $50,0 \%$ dos pacientes viram o médico, enquanto que somente $18,2 \%$ viram a enfermeira de centro cirúrgico.

A maioria $(90,9 \%)$ dos pacientes não ficaram sozinhos na sala de operaçōes; identificaram o médico $(47,7 \%)$ como a pessoa que permaneseu ao seu lado. A necessidade de segurança e bem-estar está exacerbada no período anestésico cirúrgico como declara RIDGEWAY (1976).

TABELA 6

Número e porcentagem de pacientes, sєgundo as necessidades sentidas no início de indução anestésica.

\begin{tabular}{l|c|c}
\hline NECESSIDADE SENTIDA & Número & Percentagem (\%) \\
\hline - religião & 2 & 4,5 \\
- alguém segurando a māo & 1 & 2,3 \\
- alguém para a judar a respirar & 1 & 2,3 \\
- nada & 34 & 77,3 \\
- não lembram & 6 & 13,6 \\
\hline T O T A L & 44 & 100,0 \\
\hline \hline
\end{tabular}

Observa-se que $77,3 \%$ dos pacientes nada precisavam no momento da indução anestésica.

Poucos verbalizaram sua necessidade naquele momento; aqueles que o fizeram, reivindicaram a área expressiva da assistência de enfermagem. A necessidade religiosa parece ser um apoio, como consluiu ARAÚJO (1976) em seu trabalho. O contato físico, com alguém segurando a mão, ajudando a respirar pode traduzir a necessidade de segurança.

Observa-se que $38,7 \%$ não se lembram e $20,6 \%$ dos pacientes ouviram conversas, como: discussão, gracejos.
Pode-se verificar que o paciente teve percepçōes de seu espaço - somente relatou fatos referentes à sua pessoa, como cuidados físicos, ambiente estranho, peræepçōes auditivas e mal-estar.

Do total dos pacientes, $56,8 \%$ relataram que foram cuidados na sala de recuperação pós-anestésica.

Dos resultados mencionados, parece que não há participação efetiva da enfermeira de centro cirúrgico no cuidado ao paciente, desde seu recebimen'to na unidade até o encaminhamento para a sala de recuperação pós-anestésica. 
FERRAZ, E.R. e Orientador - O Paciente Cirúrgico: suas Expectativas e Opiniōes Quanto ao Cuidado de Enfermagem no Período Transoperatório. Rev. Bras. Enf.; DF, 35 : 48-59, 1982.

\section{TABELA 7}

Número e porcentagem de pacientes, segundo os fatos de que se lembram antes de serem anestesiados.

\begin{tabular}{|c|c|c|}
\hline FATOS QUE MARCARAM & Número & Porcentagem (\%) \\
\hline $\begin{array}{l}\text { - nāo lembram } \\
\text { - ouvir conversas } \\
\text { - rezar } \\
\text { - colocação do soro } \\
\text { - presença de dores } \\
\text { - vontade de sair dali } \\
\text { - necessidade de alguém ao seu lado } \\
\text { - sensação de frio } \\
\text { - sensaçāo de perna adormecida } \\
\text { - sensação de língua grossa } \\
\text { - ouvir barulbo de caixas } \\
\text { - passagem da maca para a mesa } \\
\text { - antissepsia da pele } \\
\text { - sensação de medo }\end{array}$ & $\begin{array}{l}17 \\
9 \\
4 \\
3 \\
2 \\
2 \\
1 \\
1 \\
1 \\
1 \\
1 \\
1 \\
1 \\
1\end{array}$ & $\begin{array}{l}38,7 \\
20,6 \\
9,1 \\
6,8 \\
4,5 \\
4,5 \\
2,3 \\
2,3 \\
2,3 \\
2,3 \\
2,3 \\
2,3 \\
2,3 \\
2,3\end{array}$ \\
\hline$T O T \wedge L$ & 44 & 100,0 \\
\hline
\end{tabular}

O cuidado recebido no período transoperatório foi avaliado como "bom" por $93,2 \%$ dos pacientes.

4. Expectativas e problemas sentidos pelos pacientes, segundo as entrevistas realizadas no pré e pós-operatório.

As expectativas dos pacientes, já comentadas, não tiveram diferença significativa quanto ao sexo e idade. Quando o paciente já passou por uma experiência cirúrgica, partece que requisita maiores cuidados quanto à área expressiva (queriam gente amiga para ajudar). As expectativas se concentram na rapidez da ocorrência da anestesia e cirurgia.

Verifica-se que a preocupação com o transoperatório, que também pode ser unido ao medo do desconhecido, são os problemas mais apresentados.

A distribuição de problemas apresentados e as variáveis sexo, idade e experiência cirúrgica anterior não tiveram diferença significativa.

Avaliaram a experiência cirúrgica como excelente, porém alguns relataram-na como triste, traumatizante e dolorosa. A maioria das reclamações feitas foram compatíveis com as conseqüências de uma recuperação cirúrgica e também de uma má posição cirúrgica. 
FERRAZ, E.R. e Orientador - O Paciente Cirúrgico suas Expectativas e Opiniōes Quanto ao Cuidado de Enfermagem no Período Transoperatório. Rev. Bras. Enf.; DF, 35 : 48-59, 1982.

TABELA 8

Número e porcentagem dos pacientes, segundo problemas apresentados.

\begin{tabular}{|c|c|c|}
\hline P ROALEMAS & No & Porcentagem $(\%)$ \\
\hline $\begin{array}{l}\text { - preocupação com cuidado transoperató } \\
\text { rio }\end{array}$ & 5 & 11,4 \\
\hline - medo do desconhecido & 4 & 9.1 \\
\hline - preocupação com a familia & 3 & 6,8 \\
\hline $\begin{array}{l}\text { - preocupaçāo com possibilidade de sus } \\
\text { pensāo da cirurgia }\end{array}$ & $\mathbf{2}$ & 4,5 \\
\hline $\begin{array}{l}\text { - preocupaçāo coln cuidado pré-operató- } \\
\text { rio }\end{array}$ & 2 & 4,5 \\
\hline $\begin{array}{l}\text { - preocupaçäo com os fatos advindos da } \\
\text { cirurgia }\end{array}$ & 2 & 4,5 \\
\hline $\begin{array}{l}\text { - medo do desconhecido e preocupaçāo } \\
\text { com a familla }\end{array}$ & 2 & 4.5 \\
\hline $\begin{array}{l}\text { - preocupaçāo com quem seria o cirurgt } \\
\text { ào }\end{array}$ & 2 & 4.5 \\
\hline $\begin{array}{l}\text { - medo do desconhecido e preocupação } \\
\text { com hospitalização }\end{array}$ & 1 & 2,3 \\
\hline - nada a declarar & 21 & 47.7 \\
\hline$T \circ T \wedge L$ & 44 & 99,8 \\
\hline
\end{tabular}

\section{CONCLUSŌES}

Este estudo permitiu as seguintes conclusōes:

1. as expectativas dos pacientes, em relação ao atendimento de enfermagem no centro cirúrgico, estão relacionadas com:

- área expressiva de atuação - que tivesse gente amiga para ajudar $(6,8 \%)$;

- área instrumental ou técnica atendimento rápido para serem logo operados e anestesiados $(84,1 \%)$.

2. o atendimento de enfermagem no periodo transoperatório, segundo a opinião dos pacientes, foi relatado como:

- foram recebidos no centro cirúrgico por funcionários $(38,6 \%)$;
- foram identificados pelo nome $(77,3 \%)$;

- referiram como atividade percebida no centro cirúrgico a passagem da maca para a mesa cirúrgica $(35,8 \%)$;

- sentiram necessidade de ajuda na indução anestésica $(9,1 \%)$;

- avaliaram como "bom" o cuidado recebido $(93,2 \%)$;

3. os problemas sentidos pelos pacientes, relatados durante as entrevistas realizadas foram:

- preocupação com cuidados no período transoperatório $(11,4 \%)$;

- medo do desconhecido $(9,1 \%)$;

- preocupação com a família $(6,8 \%)$. 
FERRAZ, E.R. e Orientador - O Paciente Cirúrgico: suas Expectativas e Opiniōes Quanto ao Cuidado de Enfermagem no Período Transoperatório. Rev. Bras. Enf.; DF, 35 : 48-59, 1982.

\section{B I B LIOGR A F I A}

1. AtKinson, L. J. - The circle of patient care. AORN J., Denver, 16(3): 45-50, sept., 1972.

2. ARAÚJO, C. P. - Estudo sobre a necessidade religiosa de pacientes em fase pré-cirúrgica. São Paulo, 1976. (Dissertação de mestrado apresentada à Escola de Enfermagem da USP).

3. BALBDA, W. T. - For a better prepared patient. AANAI, Chicago, 45 (6) :621-4, dec., 1977.

4. BELAND, I. \& PASSOS, J. - Enfermagem Clinica. São Paulo, EPU/ EDUSP. 1979, v. 3, cap. 17, págs. 89-154.

5. Castellanos, B. E. P. - Aplicaçāo do processo de enfermagem ao cuidado do paciente na unidade de centro cirúrgico. Rev. Esc. Ent. USP, SP, $12(3): 170-86,1978$.

6. CASTEllanos, B. E. P. \& Ferraz, E. R. - Posição do paciente para cirurgia e implicaçōes no cuidado de enfermagem. Rev. Bras. Enf. USP, SP, 14(1):73-82, 1980.

7. CLEMONS, B. - The O, R. nurse in the patient care circuit. $A m$. J. Nurs. NY, 68(10):2141-4, oct., 1968.

8. DE LAPPE, A. - Shhh! "Sleeping". OR patients are listening. AORN J: Denver, 19(6) :1334-48, jun., 1974.

9. DUARTE, D. F. - Controle da ansiedade pré-operatória. Rev. Bras. Anest., RJ, 26(6) :815-26, dez., 1976.

10. EDWARDS, B. J. - How to talk with patient before and after surgery. AORN J., Denver, 14(5):45-9, 1971.

11. GINSBERG, F. - How OR nuses can rejoin nursing. Modern Hosp., Chicago, $112(5): 134,1969$.

12. GRAHAM, L. E. \& MYERS, E. - Evaluation of anxiety and fear in adult surgical patients. Nurs. Res., NY, 20(2) :113-23, mar.-apr., 1971.

13. GRUENDEMANN, B. J. - Analysis of the role of the professional staff nurse in operating room. Nurse. Res., NY, $19(4): 349,1971$.

14. JOHNSTON, D. F. \& HOOD, G. H. Total patient care - foundations and practice, 4 th ed. C. V. Mosby Co. Saint Louis, 1976. cap. 1, 3 e 9 .

15. JOUCLAS, V. M. G. - Elaboraçāo e avaliaçāo de um instrumento de co- municação que favoreça a assistência de enfermagem no transoperatório. São Paulo, 1977. (Dissertação de mestrado apresentada à Escola d₹ Enfermagem da USP).

16. KAMYAMA, Y. - O doente hospitalizado e sua percepçāo quanto à prioridade de seus problemas. São Paulo, 1972 (Tese de doutoramento apresentada à Escola de Enfermagem - USP)

17. KATZ, E. et alii. Pre $y$ post operatorio normal y patológico. Editorial Universitaria. Buenos Aires. v. 2, 1971, págs. 907-11.

18. LEVINE, D. C. \& FIELDLER, J. P. Fears, facts and fantasies about pre and postoperative care. Nurs Outlook, NY, 18(2): 26-8, 1970.

19. MEHAFFY, N. L. - Assessment and communication for continuity of care for the surgical patient. Nurs. Clin. North Am., Philadelphia, 10 (4) : 625-33, 1975.

20. MENEZES, A. R. - A problemática de enfermagem dos pacientes no período transoperatório - um estudo dos problemas sentidos e observados. São Paulo, 1978 (Dissertaçāo de mestrado apresentada à Escola de Enfermagem da USP).

21. PANZA, A. M. M - Efeito da visita pré-operatória da enfermeira de centro cirúrgico sobre o estresse do paciente no pré-operatório, no dia da cirurgia e no pós-operatório. São Paulo, 1977 (Dissertação de mestraclo apresentada à Escola de Enfermagem da USP).

22. RADINA, B. - What the OR nurse wants to Know. AORN J., Denver 10(2) :39, 1969.

23. RIDEGEWAY, M. - Preop interviews assure quality care. AORN J., Denver, $24(6): 1083-5,1976$.

24. RODRIGUES, A. I. $-O$ paciente no sistema centro cirúrgico. Um estudo sobre percepçōes e opiniōes de pacientes em relação ao período transoperatório. Sāo Paulo, 1979. (Dissertação de mestrado apresentada à Escola de Enfermagem da USP).

23. WELSH, J. \& RICHARDSON, A. How well do you know your patient, nurse? NAT News, Windsor, 13(5) : 12-5, 1976. 
FERRAZ, E.R. e Orientador - O Paciente Cirúrgico: suas Expectativas e Opiniōes Quanto ao Cuidado de Enferm.agem no Período Transoperatório. Rev. Bras. Enf.; DF, 35 : 48-59, 1982.

\section{ANEXO}

\section{INSTRUMENTO DE COLETA DE DADOS}

\section{I - IDENTIFICAÇÃO}

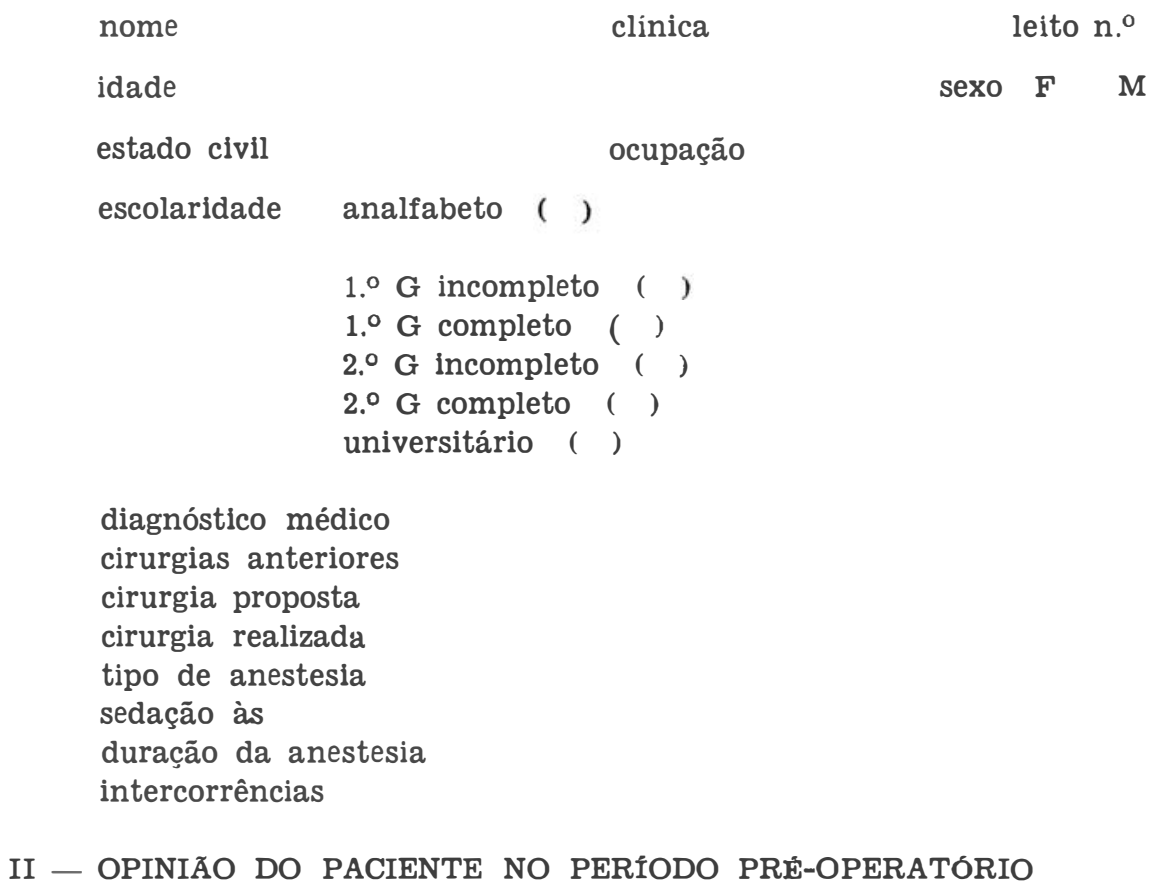

1. O (A) sr (a) sabe que vai ser operado (a)? $\operatorname{sim}($ ) não ( )

2. $\mathrm{O}(\mathrm{A})$ sr (a) sabe de que vai ser operado (a)? $\operatorname{sim}($ ) não ( )

3. O (A) sr (a) recebeu alguma explicação sobre a cirurgia?

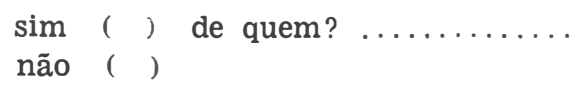

4. O (A) sr (a) tem alguma dúvida quanto à cirurgia?

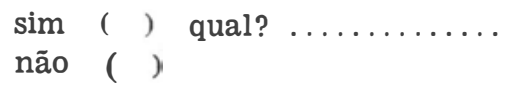

5. O (A) sr (a) já foi operado (a) anteriormente?

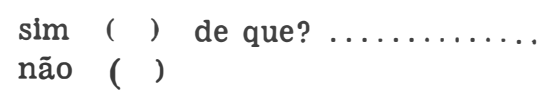

6. O (A) sr (a) conhece o centro cirúrgico? $\operatorname{sim}($ ) não ( ) 
FERRAZ, E.R. e Orientador - O Paciente Cirúrgico: suas Expectativas e Opiniōes Quanto ao Cuidado de Enfermagem no Periodo Transoperatório. Rev. Bras Enf.; DF, 35 : 48-59, 1982.

7. O (A) sr (a) conhece alguma pessoa no centro cirúrgico?

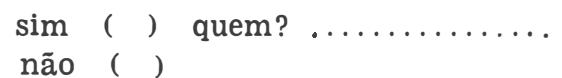

8. Chegando no centro cirúrgico, o que o (a) sr (a) espera que aconteça?

9. O (A) sr (a) gostaria que alguma pessoa permanecesse junto do (a) sr (a) enquanto iniciasse a cirurgia? $\operatorname{sim}($ ) quem? ............. não ( )

10. Gostaria de perguntar alguma coisa?

11. O (A) sr (a) conhece a enfermeira desta clínica? $\left.\operatorname{sim}_{\text {não }}()^{\prime}\right)$ quem é? $\ldots \ldots \ldots \ldots \ldots$

Observaçōes -

inicio:

término:

\section{III - OPINIÃO COLHIDA NO PERÍODO PÓS-OPERATÓRIO}

1. Chegando ao centro cirúrgico, quem o recebeu?

2. Permaneceu algum tempo no corredor?

$\operatorname{sim}($ ) como se sentiu?

(não ( )

3. Durante sua permanência no corredor, alguém lhe deu atenção?

$\operatorname{sim}($ ) quem? $\ldots \ldots \ldots \ldots \ldots$

não ( )

4. Como foi identificado (a)?

nome ( )

n. ${ }^{\circ}$ da sala de operação ( )

nome do cirurgião ( )

nome da cirurgia ( )

outro ( ) ...............

5. Chegando na sala de operaçōes, o que fizeram com a (o) sr (a)?

6. Quem o (a) ajudou a passar da maca para a mesa cirúrgica?

7. O (A) sr (a) viu o médico antes de ser operado (a)? $\operatorname{sim}($ ) não ( )

8. O (A) sr (a) viu quem é a enfermeira do centro cirúrgico? $\operatorname{sim}$ ( ) o que ela fez pelo (a) sr (a) ? não ( ) 
FERRAZ, E.R. e Orientador - O Paciente Cirúrgico: suas Expectativas e Opiniōes Quanto ao Cuidado de Enfermagem no Periodo Transoperatório. Rev. Bras. Enf.; DF, 35 : 48-59, 1982.

9. O (A) sr (a) ficou sozinho (a) na sala de operaçōes? $\operatorname{sim}($ ) como se sentiu? não ( )

10. Quem permaneceu mais tempo ao seu lado?

11. Durante a fase de anestesia, como se sentiu?

12. Durante essa fase, o (a) sr (a) precisava de alguma coisa?

13. Qual foi a última coisa que o (a) sr (a) lembra no momento em que estava sendo anestesiado (a)?

14. O (A) sr (a) lembra do que ouviu quando despertou da anestesia?

15. O (A) sr (a) foi para a sala de recuperação pós-anestésica? $\operatorname{sim}($ ) a) como foi o (a) sr (a) tratado (a)?
não ( ) o (a) sr (a) conheceu a enfermeira?

16. O (A) sr (a) foi cuidado, no centro cirúrgico como esperava? $\operatorname{sim}($ ) não ( ) como gostaria que ocorresse?

17. O que o (a) sr (a) gostaria que tivesse sido feito durante sua permanência no centro cirúrgico?

17. O que o (a) sr (a) achou dessa experiência cirúrgica?

19. O (A) sr (a) ouviu algum barulho ou conversa que o (a) desagradou?

20. O (A) sr (a) teria alguma reclamação ou algo a dizer?

Observaçōes

início:

término: 